\title{
Concordancia entre los modelos de SCORE y Framingham y las ecuaciones AHA/ACC como evaluadores de riesgo cardiovascular
}

\author{
Oscar Mauricio Muñoz V ${ }^{a, *}$, Álvaro J. Ruiz Morales ${ }^{b}$, Alejandro Mariño Correa ${ }^{a}$ \\ y Marlon Mauricio Bustos C. ${ }^{a}$
}

\author{
a Departamento de Medicina Interna, Pontificia Universidad Javeriana, Hospital Universitario San Ignacio, Bogotá, Colombia \\ b Departamento de Epidemiología Clínica y Bioestadística, Pontificia Universidad Javeriana, Hospital Universitario San Ignacio, \\ Bogotá, Colombia
}

Recibido el 18 de enero de 2016; aceptado el 8 de junio de 2016

Disponible en Internet el 21 de septiembre de 2016

\author{
PALABRAS CLAVE \\ Epidemiología; \\ Prevención primaria; \\ Enfermedad \\ cardiovascular; \\ Riesgo de \\ enfermedad \\ coronaria; \\ Estudios de \\ concordancia
}

\begin{abstract}
Resumen
Objetivo: Diferentes modelos de evaluación de riesgo cardiovascular están actualmente en uso en Colombia. El objetivo de este estudio es analizar la concordancia entre las ecuaciones AHA/ACC 2013, SCORE y Framingham ajustado, así como el impacto de usar una u otra en la cantidad de pacientes clasificados como de alto riesgo y en la cantidad de pacientes que requerirían manejo farmacológico.

Métodos: Se evaluaron 800 pacientes entre 40 y 74 años, de la clínica de prevención primaria del Hospital militar Central en Bogotá (Colombia), libres de eventos cardiovasculares. Se estimaron el riesgo a 10 años de enfermedad arterioesclerótica cardiovascular según las ecuaciones propuestas por las guías AHA/ACC 2013, el riesgo de muerte cardiovascular según la función SCORE de la guía europea y el riesgo coronario según la función de Framingham ajustada, recomendada por la guía colombiana. Se consideró como indicación de manejo farmacológico un cálculo de riesgo AHA/ACC o Framingham ajustado $>7,5 \%$. Un riesgo de Framingham $>20 \%$ o SCORE > 5\% definía el riesgo alto.

Resultados: Según el Framingham había un 5,9\% de pacientes de alto riesgo, según las ecuaciones de SCORE para países de bajo riesgo un 18,7\% y según las ecuaciones de SCORE para países de alto riesgo, un 31,2\%. El coeficiente Kappa mostró baja concordancia entre Framingham ajustado y cada una de las ecuaciones de SCORE (0,28 y 0,22 respectivamente). Según las recomendaciones de la guía $\mathrm{AHA} / \mathrm{ACC}$, el tratamiento hipolipemiante estaría indicado en un $40,8 \%$ de los pacientes, frente a un $50,6 \%$ según la guía colombiana (Framingham ajustado). El coeficiente kappa fue de 0,5735.
\end{abstract}

\footnotetext{
* Autor para correspondencia.

Correos electrónicos: o.munoz@javeriana.edu.co, o.munozv@yahoo.com (O.M. Muñoz V).
} 
Conclusiones: En la actualidad existe pobre acuerdo entre las diferentes escalas de evaluación del riesgo cardiovascular usadas en Colombia, hecho que conlleva incertidumbre para la toma de decisiones terapéuticas. Los datos de este estudio demuestran la necesidad de validar los modelos de SCORE y AHA/ACC en Colombia y Latinoamérica.

(c) 2016 Sociedad Colombiana de Cardiología y Cirugía Cardiovascular. Publicado por Elsevier España, S.L.U. Este es un artículo Open Access bajo la licencia CC BY-NC-ND (http:// creativecommons.org/licenses/by-nc-nd/4.0/).

\section{KEYWORDS}

Epidemiology; Primary prevention; Cardiovascular diseases; Risk of coronary heart disease; Concordance studies
Concordance between the SCORE and Framingham models and ACC/AHA equations as cardiovascular risk indicators

\begin{abstract}
Motivation: In Colombia, different models of cardiovascular risk assessment are currently being used. The motivation of this study is to analyse the concordance between the ACC/AHA 2013 equation, SCORE and adjusted Framingham, as well as the impact of using one or another in the amount of patients classified as high risk and the amount of patients requiring pharmacological management.

Methods: 800 patients between 40 and 74 years old were assessed, from the primary prevention clinic of the Hospital Militar Central in Bogotá (Colombia), who were free of cardiovascular events. 10-year risk for atherosclerotic vascular disease was estimated according to the equations proposed by ACC / AHA 2013 guides, the risk of cardiovascular death according to the SCORE function of the European guide and the coronary risk according to the adjusted Framingham function recommended by the Colombian guide. The indication of pharmacological management was considered with an ACC/AHA or adjusted Framingham risk of $>7.5 \%$. A $>20 \%$ Framingham or a $>5 \%$ SCORE risk were considered high risk.

Results: According to Framingham there was a $5.9 \%$ of high-risk patients, according to SCORE equations for low-risk countries an $18.7 \%$ and according to SCORE equations for high-risk countries, a 31.2\%. The Kappa coefficient showed a low concordance between adjusted Framingham and each of the SCORE equations ( 0.28 and 0.22 respectively). According to the ACC/AHA guide recommendations, hypolipidemic treatment would be indicated in $40.8 \%$ of patients, versus a $50.6 \%$ following the Colombian guide indications (adjusted Framingham). Kappa coefficient was 0.5735 .

Conclusions: Nowadays there is a poor agreement between the different cardiovascular risk assessment scales used in Colombia, thus generating uncertainty when it comes to making therapeutic choices. Data from this study show the need to validate the validate the SCORE and ACC/AHA models in Colombia and Latin America.

(c) 2016 Sociedad Colombiana de Cardiología y Cirugía Cardiovascular. Published by Elsevier España, S.L.U. This is an open access article under the CC BY-NC-ND license (http:// creativecommons.org/licenses/by-nc-nd/4.0/).
\end{abstract}

\section{Introducción}

La evaluación individual del riesgo cardiovascular para cada paciente, y el tratamiento acorde con ese riesgo, se han convertido en el estándar de cuidado recomendado por las diferentes guías de manejo del mundo ${ }^{1-3}$. En la actualidad se dispone de múltiples modelos matemáticos que estiman el riesgo cardiovascular con base en los factores de riesgo de cada individuo. Los tres sistemas más aceptados como estimadores de riesgo cardiovascular en Colombia incluyen las ecuaciones recomendadas por la guía de la Asociación americana del corazón y el Colegio americano de cardiología (ACC/AHA, sus siglas en inglés) del año 2013 ${ }^{1}$, el modelo SCORE (Systematic Coronary Risk Evaluation) propuesto por la guía europea ${ }^{2}$ y la escala de Framingham ajustada (riesgo cardiovascular Framingham $x 0,75)$ propuesta recientemente por la Guía de práctica clínica para la prevención, detección temprana, diagnóstico, tratamiento y seguimiento de las dislipidemias en Colombia ${ }^{3}$.

Cada una de estas escalas tiene ventajas y desventajas para ser utilizada como estimador de riesgo cardiovascular en Colombia. Las ecuaciones AHA/ACC de 2013 tienen como ventaja que estiman de manera simultánea el riesgo de desarrollar eventos cardiovasculares y cerebrovasculares duros a 10 años (incluyendo muerte de origen coronario, infarto agudo de miocardio no fatal y ataque cerebrovascular fatal o no fatal) todos ellos desenlaces relevantes tanto para los médicos como para los pacientes. A este riesgo lo denominaron enfermedad aterosclerótica cardiovascular. Su principal limitación está dada por la imposibilidad para generalizar las ecuaciones a la población colombiana, dado que las cohortes sobre las que se desarrolló este modelo estaban compuestas fundamentalmente por poblaciones 
de norteamericanos blancos y afrodescendientes. Más aún, su grupo desarrollador declaró abiertamente que la representación de otros grupos étnicos fue insuficiente, de modo que recomiendan realizar estudios de validación externa para evaluar el uso de estas nuevas ecuaciones por fuera de la población en la que fue desarrollada ${ }^{1}$.

Una segunda opción es utilizar el modelo SCORE ${ }^{4}$, recomendado por la Guía de la Sociedad europea de cardiología y otras nueve sociedades relacionadas con el manejo del riesgo cardiovascular ${ }^{2}$. Este modelo estima exclusivamente el riesgo de muerte a 10 años, bien sea por enfermedad isquémica del corazón, enfermedad cerebrovascular o arteriopatía periférica. Sus autores proponen que evaluar exclusivamente mortalidad resulta ventajoso, dado que quienes tienen alto riesgo de morir también tienen alto riesgo de presentar otros eventos cardio o cerebrovasculares. Adicionalmente, proponen que utilizar desenlaces duros hace más fácil validar y recalibrar el modelo, facilitando su utilización en diferentes entornos ${ }^{2}$, como en efecto se ha hecho en distintos países europeos, donde se pudieron desarrollar escalas diferenciales para países de alto y bajo riesgo ${ }^{4}$. Su principal desventaja radica nuevamente en que no ha sido validada en Colombia.

Recientemente, el Ministerio de salud y Protección social de Colombia y COLCIENCIAS patrocinaron el desarrollo de la Guía de práctica clínica para la prevención, detección temprana, diagnóstico, tratamiento y seguimiento de las dislipidemias en Colombia ${ }^{3}$.

Esta guía propone utilizar la escala de Framingham como estimador del riesgo cardiovascular en Colombia, pero ajustando el riesgo por un factor de corrección de 0,75. Este ajuste se basa en un estudio que demostró que la escala de Framingham sobreestima el riesgo cardiovascular en Colombia en un $30 \%^{5}$. Las ventajas propuestas por la Guía colombiana incluyen que la escala de Framingham está validada en Colombia ${ }^{5}$ y es ampliamente conocida y utilizada por los médicos colombianos.

Utilizar simultáneamente en el país varios modelos para calcular el riesgo cardiovascular tiene como desventaja que los pacientes pueden quedar clasificados de forma diferente por cada escala. Adicionalmente, la cantidad de pacientes que se debe tratar puede ser diferente de acuerdo con la escala que se utilice. El presente estudio evalúa la concordancia entre los diferentes modelos para calcular riesgo cardiovascular en una población colombiana y examina sus implicaciones terapéuticas.

\section{Métodos}

Se utilizó como población de estudio a todos pacientes que ingresaron a la Clínica de Prevención Primaria del Hospital Militar Central (Bogotá, Colombia) entre 1984 y 1996. Los datos de ingreso al programa se tabularon como un estudio de corte transversal. Las características de esta población ya han sido descritas en un estudio previo ${ }^{5}$. Se seleccionaron los pacientes que cumplieron los criterios de inclusión: edad entre 40 y 74 años (rango de edad aceptado para evaluar el riesgo cardiovascular por los tres modelos en estudio) y estar libres de enfermedad cardiovascular cuando ingresaron al programa. Se consideraron libres de enfermedad cardiovascular si a su ingreso a la clínica de prevención primaria no reportaban infarto de miocardio o evento cerebrovascular diagnosticados por un médico, y no presentaban evidencia de infarto agudo de miocardio en electrocardiograma de 12 derivaciones de ingreso, definido como la presencia de onda Q significativa (mayor del $30 \%$ de la amplitud total del QRS).

Se extrajeron los datos relativos a las variables de exposición registradas al ingreso de los pacientes a la clínica de prevención primaria, incluyendo la información de edad, sexo, índice de masa corporal (IMC), glucemia en ayunas y uso de hipoglucemiantes, tensión arterial sistólica, diastólica y uso de antihipertensivos, tabaquismo, antecedentes familiares, colesterol total, colesterol-LDL, colesterol-HDL y triglicéridos. Cuando no se realizó medición directa de colesterol LDL se calculó el mismo mediante la fórmula de Friedewald $^{6}$.

Con base en estos datos se calculó el riesgo de presentar enfermedad coronaria total a 10 años (muerte de origen coronario, infarto del miocardio, angina de pecho o insuficiencia coronaria) de acuerdo con la escala de predicción de riesgo de Framingham ${ }^{7}$ usando las definiciones operativas y categorías que se emplearon en el estudio publicado originalmente. El resultado se multiplicó por 0,75 de acuerdo con lo recomendado en la Guía colombiana ${ }^{3}$. Se clasificaron como pacientes de alto riesgo a aquellos que según el modelo de Framingham ajustado tuvieran un cálculo de riesgo cardiovascular superior al 20\%. Adicionalmente, se consideró que tenían indicación de tratamiento farmacológico con estatinas aquellos en quienes el cálculo de riesgo cardiovascular con el modelo de Framingham ajustado fuera superior a 7,5\%, de acuerdo con lo recomendado en las guías colombianas $^{8}$.

De igual forma se calculó el riesgo de mortalidad cardiovascular con base en las ecuaciones propuestas por SCORE ${ }^{4}$. Considerando que la escala no ha sido validada en Colombia se realizaron los cálculos tanto para las funciones de países de bajo como de alto riesgo. Se clasificaron como pacientes de alto riesgo a aquellos en quienes el cálculo de riesgo de mortalidad cardiovascular a 10 años fuese superior al 5\% (equivalente a la clasificación actual de pacientes con riesgo aumentado).

Por último, se calculó el riesgo de enfermedad aterosclerótica cardiovascular de acuerdo con las ecuaciones propuestas por la AHA/ACC 2013, disponibles en http://my.americanheart.org/cvriskcalculator y en http://www.cardiosource.org/en/Science-And-Quality/ Practice-Guidelines-and-Quality-Standards/2013-Prevention -Guideline-Tools.aspx. Según la recomendación expresada en esa misma guía se utilizaron las fórmulas diseñadas para pacientes norteamericanos blancos no hispánicos. Se consideró que tenían indicación de tratamiento farmacológico con estatinas aquellos en quienes el cálculo de riesgo de enfermedad aterosclerótica cardiovascular fuera superior a 7,5\%. También se hizo el cálculo de la cantidad de pacientes con riesgo mayor de $5 \%$, considerando que la guía sugiere que podrían requerir manejo farmacológico si tuviesen asociados otros factores de riesgo adicionales (LDL $>160$, antecedente familiar de enfermedad aterosclerótica prematura, PCR-us $\geq 2,0 \mathrm{mg} / \mathrm{L}$, índice de calcio en las arterias coronarias $\geq 300$ unidades Agaston o índice tobillo brazo $<0,9)$. 
Tabla 1 Características demográficas de la población

\begin{tabular}{|c|c|c|c|}
\hline & $\begin{array}{l}\text { Hombres } \\
(\mathrm{n}=287)\end{array}$ & $\begin{array}{l}\text { Mujeres } \\
(\mathrm{n}=513)\end{array}$ & $\begin{array}{l}\text { Global } \\
(n=800)\end{array}$ \\
\hline Edad (años)(Media DE) & 56,5 DE 7,87 & 54,1 DE 8,02 & 54,9 DE 8,05 \\
\hline Diabetes & $14(4,9 \%)$ & $31(6,0 \%)$ & $45(5,6 \%)$ \\
\hline \multicolumn{4}{|l|}{ Presión arterial } \\
\hline Hipertensión & $231(80,5 \%)$ & $350(68,2 \%)$ & $581(72,6 \%)$ \\
\hline \multicolumn{4}{|l|}{ Colesterol total } \\
\hline$>200 \mathrm{mg} / \mathrm{dl}$ & $229(79,8 \%)$ & $404(78,8 \%)$ & $633(79,1 \%)$ \\
\hline \multicolumn{4}{|l|}{ Colesterol LDL } \\
\hline$>130 \mathrm{mg} / \mathrm{dl}$ & $178(62,0 \%)$ & $330(64,3 \%)$ & $508(63,6 \%)$ \\
\hline \multicolumn{4}{|l|}{ Triglicéridos } \\
\hline$>150 \mathrm{mg} / \mathrm{dl}$ & $146(50,9 \%)$ & $204(39,8 \%)$ & $350(43,8 \%)$ \\
\hline \multicolumn{4}{|l|}{ Colesterol HDL } \\
\hline$<40 \mathrm{mg} / \mathrm{dl}$ & $43(15,0 \%)$ & $52(10,1 \%)$ & $95(11,9 \%)$ \\
\hline$I M C>25$ & $207(72,1 \%)$ & $381(74,3 \%)$ & $588(73,5 \%)$ \\
\hline Tabaquismo & $27(9,4 \%)$ & $41(7,9 \%)$ & $68(8,5 \%)$ \\
\hline Antecedentes familiares & $64(22,3 \%)$ & $134(26,1 \%)$ & $198(24,8 \%)$ \\
\hline
\end{tabular}

\section{Análisis estadístico}

Las variables continuas se expresan como media y la medida de dispersión como desviación estándar si siguen una distribución normal, y como mediana e intervalo intercuartílico (RIC) si no lo hacen. Las variables categóricas se expresan como porcentajes.

Para analizar la concordancia entre los diferentes métodos de clasificación se utilizó el índice kappa. Se evaluó la concordancia entre los pacientes clasificados como de alto riesgo según Framingham ajustado y SCORE, así como la concordancia entre los pacientes con indicación de manejo farmacológico según Framingham ajustado y las ecuaciones AHA/ACC. Se fijó un nivel de significación alfa de 0,05. Con los pacientes disponibles para el análisis se calculó un poder mayor al 95\%. Los cálculos se realizaron con ayuda del programa estadístico STATA 11.

\section{Resultados}

Se evaluaron inicialmente las historias clínicas de 1.036 pacientes, de los cuales se tuvieron en cuenta para el análisis 800 , que cumplieron con los criterios de inclusión.

Las características demográficas de la población diferenciada por sexo se presentan en la tabla 1. La mayoría de los pacientes fueron de sexo femenino $(n=513,64,9 \%)$. Llama la atención una mayor frecuencia de hipertensión arterial, hipertrigliceridemia, HDL bajo y tabaquismo en los hombres.

El porcentaje de pacientes que según el Framingham ajustado fueron clasificados como de riesgo alto (> 20\%) alcanzó un $5,9 \%$ de la población, comparado con un riesgo alto según SCORE (> 5\%) de $18,7 \%$ cuando se utilizaron las ecuaciones para países de riesgo bajo, y de $31,2 \%$ cuando se utilizaron las ecuaciones para países de riesgo alto (tabla 2).
El coeficiente kappa de concordancia para clasificar a los pacientes de riesgo alto entre Framingham ajustado y SCORE para países de bajo riesgo fue de 0,2808 (IC 95\% 0,2519$0,3097)$. Los pacientes concordantes de alto riesgo fueron 34 (4,3\% del total). 129 casos fueron discordantes, de ellos 13 pertenecían al grupo con riesgo alto para Framingham y no alto según SCORE, y 116 fueron clasificados como riesgo cardiovascular alto SCORE y no alto Framingham (tabla 3).

El coeficiente kappa para clasificar a los pacientes de riesgo alto entre Framingham ajustado y SCORE para países de alto riesgo fue de 0,2265 (IC 95\% 0,2035-0,2495). Los pacientes concordantes de alto riesgo fueron 45 (5,6\%

Tabla 2 Riesgo cardiovascular según los modelos de Framingham ajustado, SCORE y las ecuaciones AHA/ACC

\begin{tabular}{lc}
\hline & Global $(\mathrm{n}=800)$ \\
\hline $\begin{array}{l}\text { Riesgo según Framingham ajustado } \\
\text { Bajo }<7,5 \%\end{array}$ & $395(49,4 \%)$ \\
Intermedio 7,5 a 20\% & $358(44,7 \%)$ \\
$\quad$ Alto > 20\% & $47(5,9 \%)$ \\
Riesgo según SCORE & \\
(países de alto riesgo) & \\
Bajo $<1 \%$ & $239(29,9 \%)$ \\
Intermedio 1 a 5\% & $311(38,9 \%)$ \\
Alto > 5\% & $250(31,2 \%)$ \\
Riesgo según SCORE & \\
(países de bajo riesgo) & \\
Bajo < 1\% & \\
Intermedio 1 a 5\% & $340(42,5 \%)$ \\
Alto > 5\% & $310(38,8 \%)$ \\
Riesgo según ecuaciones & $150(18,7 \%)$ \\
AHA/ACC 2013 & \\
$>7,5 \%$ & \\
\hline
\end{tabular}


Tabla 3 Tabla de concordancia de pacientes con riesgo alto según la ecuación de Framingham ajustado y SCORE (ecuaciones para países de bajo riesgo)

\begin{tabular}{|c|c|c|c|}
\hline & \multicolumn{2}{|c|}{ SCORE } & \multirow[t]{2}{*}{ Total } \\
\hline & No alto & Alto & \\
\hline \multicolumn{4}{|c|}{ Framingham ajustado } \\
\hline \multicolumn{4}{|c|}{ No alto } \\
\hline Recuento & 637 & 116 & 753 \\
\hline$\%$ del total & $79,6 \%$ & $14,5 \%$ & $94,1 \%$ \\
\hline \multicolumn{4}{|l|}{ Alto } \\
\hline Recuento & 13 & 34 & 47 \\
\hline$\%$ del total & $1,6 \%$ & $4,3 \%$ & $5,9 \%$ \\
\hline \multicolumn{4}{|l|}{ Total } \\
\hline Recuento & 650 & 150 & 800 \\
\hline$\%$ del total & $81,2 \%$ & $18,8 \%$ & $100 \%$ \\
\hline
\end{tabular}

Kappa = 0,2808 (IC 95\% 0,2519-0,3097). Riesgo alto Framingham ajustado: riesgo $\geq 20 \%$ a los 10 años. Riesgo alto SCORE: riesgo $\geq 5 \%$ a los 10 años.

del total). De los 207 casos discordantes (25,9\% del total), la mayoría (205 casos) pertenecía al grupo con riesgo alto SCORE y no alto Framingham (tabla 4).

El porcentaje de pacientes que según el Framingham ajustado requerirían manejo farmacológico (> 7,5\%) alcanzó un $50,6 \%$ de la población, comparado con un $40,8 \%$ según las ecuaciones AHA/ACC (tabla 5). La evaluación de concordancia demostró un kappa de 0,5735 (IC 95\% 0,5388-0,6082). Los pacientes concordantes en la indicación de manejo farmacológico fueron 280 (35\% del total). De los 171 casos discordantes, 125 requerirían manejo según Framingham, pero no según $\mathrm{AHA} / \mathrm{ACC}$, y 46 requerirían manejo según AHA/ACC pero no según Framingham (tabla 5 ). Si se consideran como candidatos a manejo farmacológico aquellos con riesgo $\mathrm{AHA} / \mathrm{ACC}>5 \%$, el porcentaje de pacientes que se debería tratar sería $55,5 \%$ y la concordancia con el modelo de Framingham ajustado mejoría discretamente, con un coeficiente kappa 0,6270 (IC 95\% 0,5918-0,6622).

Tabla 4 Tabla de concordancia de pacientes con riesgo alto según la ecuación de Framingham ajustado y SCORE (ecuaciones para países de alto riesgo)

\begin{tabular}{llll}
\hline & \multicolumn{2}{c}{ SCORE } & Total \\
\cline { 2 - 3 } & No alto & Alto & \\
\hline $\begin{array}{l}\text { Framingham ajustado } \\
\text { No alto }\end{array}$ & & & \\
$\quad \begin{array}{l}\text { Recuento } \\
\quad \% \text { del total }\end{array}$ & 548 & 205 & 753 \\
$\begin{array}{l}\text { Alto } \\
\quad \text { Recuento }\end{array}$ & $68,5 \%$ & $25,6 \%$ & $84,1 \%$ \\
$\quad \%$ del total & $0,3 \%$ & & \\
$\begin{array}{l}\text { Total } \\
\text { Recuento } \\
\% \text { del total }\end{array}$ & 550 & $5,6 \%$ & $5,9 \%$ \\
\hline
\end{tabular}

Kappa = 0,2265 (IC 95\% 0,2035-0,2495). Riesgo alto Framingham ajustado: riesgo $\geq 20 \%$ a los 10 años. Riesgo alto SCORE: riesgo $\geq 5 \%$ a los 10 años.
Tabla 5 Tabla de concordancia de pacientes con indicación de manejo farmacológico según Framingham ajustado y las ecuaciones AHA/ACC

\begin{tabular}{|c|c|c|c|}
\hline & \multicolumn{2}{|c|}{ Ecuaciones AHA/ACC } & \multirow[t]{2}{*}{ TOTAL } \\
\hline & Manejo & No manejo & \\
\hline \multirow{2}{*}{\multicolumn{4}{|c|}{$\begin{array}{c}\text { Framingham ajustado } \\
\text { Requiere manejo } \\
\text { farmacológico }\end{array}$}} \\
\hline & & & \\
\hline Recuento & 280 & 125 & 405 \\
\hline$\%$ del total & $35 \%$ & $15,6 \%$ & $50,6 \%$ \\
\hline \multicolumn{4}{|c|}{$\begin{array}{l}\text { No requiere } \\
\text { manejo } \\
\text { farmacológico }\end{array}$} \\
\hline Recuento & 46 & 349 & 395 \\
\hline$\%$ del total & $5,8 \%$ & $43,6 \%$ & $49,4 \%$ \\
\hline \multicolumn{4}{|l|}{ Total } \\
\hline Recuento & 326 & 474 & 800 \\
\hline$\%$ del total & $40,8 \%$ & $59,2 \%$ & $100 \%$ \\
\hline
\end{tabular}

Kappa =0,5735 (IC 95\% 0,5388-0,6082). Manejo farmacológico indicado según Framingham ajustado: riesgo $\geq 7,5 \%$ a los 10 años. Manejo farmacológico indicado según AHA/ACC: riesgo $\geq$ $7,5 \%$ a los 10 años.

\section{Discusión}

Este es el primer estudio que compara los tres métodos de evaluación de riesgo cardiovascular más aceptados actualmente, en una población colombiana. Nuestra población objeto de estudio incluye pacientes procedentes de todas las regiones del país y ha demostrado tener un perfil epidemiológico similar al reportado para toda la población del país en las primeras causas de mortalidad y morbilidad identificadas por el Ministerio de la Protección Social, característica que la convierte en una buena representación de cómo se comportarían los diferentes modelos en Colombia ${ }^{9}$. Se documentó baja concordancia entre las diferentes ecuaciones evaluadas como estimadores de riesgo cardiovascular a 10 años, tanto para definir los pacientes que resultarían clasificados como de alto riesgo, como para determinar aquellos que requerirían manejo farmacológico con estatinas. Este hallazgo conlleva implicaciones tanto clínicas como de salud pública.

Desde el punto de vista clínico un porcentaje importante de pacientes tendrían clasificaciones de riesgo distintas, hecho que puede generar dificultades para los clínicos al momento de definir un manejo adecuado para cada paciente. Este estudio demuestra que entre el 16 y el $25 \%$ de los pacientes tienen clasificaciones de riesgo discordantes al comparar el modelo de Framingham ajustado para Colombia con las diferentes ecuaciones de SCORE, siendo la concordancia discretamente mejor al utilizar las ecuaciones de SCORE para países de bajo riesgo. De igual forma, un $21,4 \%$ de los pacientes tuvieron clasificaciones diferentes al comparar el Framingham ajustado para Colombia con las ecuaciones AHA/ACC 2013 al definir quienes requerirían manejo farmacológico con estatinas.

Algunos estudios previos han demostrado que al aplicar diferentes modelos de predicción de riesgo cardiovascular en un mismo grupo de pacientes, es posible generar 
clasificaciones diferentes en cerca del $30 \%$ de los $\operatorname{casos}^{10}$. Específicamente, al comparar Framingham con SCORE, los estudios realizados en Europa han encontrado hallazgos diferentes en cuanto al grado entre las dos clasificaciones, con algunos que demuestran una concordancia muy baja ${ }^{11,12}$, y otros que han encontrado concordancias moderadas ${ }^{13}$. Los estudios de validación, sin embargo, han demostrado que las ecuaciones de SCORE son mejores a la hora de predecir el riesgo en España ${ }^{14}$ y Francia $^{15}$, contrario a lo encontrado en poblaciones de Australia, Nueva Zelanda y Reino Unido donde la escala de Framingham tiene un mejor desempeño ${ }^{16}$. En el ámbito latinoamericano, solo un estudio desarrollado en Méjico evaluó la concordancia entre las escalas de Framingham y PROCAM ${ }^{17}$, y encontró una alta proporción de pacientes que recibieron clasificaciones discordantes, especialmente en aquellos de riesgo moderado y alto, hallazgos similares a los encontrados en el presente estudio. Ningún estudio hasta ahora ha validado las ecuaciones de SCORE en poblaciones latinoamericanas, y sólo uno realizado en Colombia ${ }^{5}$ evaluó el desempeño del modelo de Framingham, que sobreestima de forma significativa el riesgo cardiovascular (relación entre eventos esperados y observados de 1,31).

Algunos estudios recientes han evaluado el desempeño de las nuevas ecuaciones de la AHA/ACC 2013 en diferentes poblaciones, y sugieren que tienen un buen desempeño en Estados Unidos ${ }^{18}$, pero pobre calibración y capacidad de discriminación en poblaciones externas a aquellas en donde fueron desarrolladas ${ }^{19,20}$. En Latinoamérica un estudio reciente evaluó la concordancia entre las nuevas ecuaciones AHA/ACC y seis diferentes escalas de estimación de riesgo cardiovascular, entre ellas SCORE y Framingham ${ }^{21}$, y encontró baja concordancia entre ellas (kappa 0,22 y 0,33 respectivamente).

En nuestro estudio, un 40,8\% de los pacientes fueron candidatos a manejo farmacológico con estatinas de acuerdo con la escala AHA/ACC, a diferencia de un 50,6\% que lo requerirían de acuerdo con la escala de Framingham ajustada. Estos resultados van en contra de lo planteado por los desarrolladores de la escala AHA/ACC, quienes esperaban que esta escala sobreestimara el riesgo cardiovascular en poblaciones hispanas ${ }^{22}$. Si se incluyen como candidatos a manejo farmacológico aquellos con riesgo enfermedad cardiovascular ateroesclerótica 5\%, como lo indica la guía para pacientes que además de este valor tengan algún otro factor de riesgo, el porcentaje de pacientes que se debe tratar sería similar (50,6 vs. 55,5\%) y el valor de kappa demostraría una buena concordancia $(0,6270)$. El impacto económico de usar una u otra escala para clasificar a los pacientes, está por determinarse.

Adicionalmente, nuestros hallazgos son importantes dado que demuestran una utilidad limitada de los modelos de predicción de riesgo generados en otros países, tanto para hacer planeación en nuestro sistema de salud como para tomar medidas que tengan verdadero impacto en cuanto a salud pública. La mejor forma de afrontar esta dificultad es validar cualquier modelo que se quiera usar en Colombia antes de aplicarlo en nuestros pacientes y por ahora utilizar aquellos que ya han sido validados y recalibrados en el país, como fue la decisión tomada por la Guía colombiana para la prevención, detección temprana, diagnóstico, tratamiento y seguimiento de las dislipidemias ${ }^{3}$.

\section{Conclusiones}

Los resultados de este estudio sugieren un pobre acuerdo entre las diferentes escalas de evaluación del riesgo cardiovascular actualmente usadas en Colombia, siendo mejor la concordancia entre AHA/ACC y Framingham ajustado, que entre esta última y SCORE. Estos hallazgos dan cuenta de la incertidumbre a la hora de elegir una de estas herramientas tanto para tomar decisiones clínicas para el manejo individual de cada paciente, como para tomar decisiones de salud pública. Los datos obtenidos demuestran la necesidad de validar cualquier nuevo modelo que se quiera usar localmente antes incluirlo en la práctica diaria e invitan a preferir el uso de aquellos que ya han sido validados y recalibrados en Colombia.

\section{Responsabilidades éticas}

Protección de personas y animales. Los autores declaran que para esta investigación no se han realizado experimentos en seres humanos ni en animales.

Confidencialidad de los datos. Los autores declaran que en este artículo no aparecen datos de pacientes.

Derecho a la privacidad y consentimiento informado. Los autores declaran que en este artículo no aparecen datos de pacientes.

\section{Conflictos de interés}

Los autores declaran no tener conflictos de interés.

\section{Bibliografía}

1. Goff DC, Lloyd-Jones DM, Bennett G, O’Donnell CJ, Coady S, Robinson J, et al. 2013 ACC/AHA Guideline on the Assessment of Cardiovascular Risk: A Report of the American College of Cardiology/American Heart Association Task Force on Practice Guidelines. Circulation. 2014;129 25 Suppl 2:s49-73.

2. Perk J, De Backer G, Gohlke H, Graham I, Reiner Z, Verschuren $M$, et al. European Guidelines on cardiovascular disease prevention in clinical practice (version 2012): The Fifth Joint Task Force of the European Society of Cardiology and Other Societies on Cardiovascular Disease Prevention in Clinical Practice. Eur Heart J. 2012;33:1635-701.

3. Muñoz OM, García AA, Fernández-Ávila D, Higuera A, Ruiz AJ, Ascher P. Guía de práctica clínica para la prevención, detección temprana, diagnóstico, tratamiento y seguimiento de las dislipidemias: evaluación del riesgo cardiovascular. Rev Colomb Cardiol. 2015;22:263-9.

4. Conroy R, Pyorala K, Fitzgerald A, Sans S. Estimation of tenyear risk of fatal cardiovascular disease in Europe: the SCORE project. Eur Hear J. 2003;24:987-1003.

5. Muñoz OM, Rodríguez NI, Ruiz Á, Rondón M. Validación de los modelos de predicción de Framingham y PROCAM como estimadores del riesgo cardiovascular en una población colombiana. Rev Colomb Cardiol. 2014;21:202-12.

6. Friedewald W, Levy R, Fredrickson D. Estimation of the concentration of low-density lipoprotein cholesterol in plasma, without use of the preparative ultracentrifuge. Clin Chem. 1972;18:499-502. 
7. Executive Summary of The Third Report of The National Cholesterol Education Program (NCEP) Expert Panel on Detection, Evaluation, And Treatment of High Blood Cholesterol In Adults (Adult Treatment Panel III). JAMA. 2001; 285:2486-97.

8. Muñoz 0 , García A, Fernández D, Higuera A, Ruiz A, Aschner P, et al. Guía de práctica clínica para la prevención, detección temprana, diagnóstico, tratamiento y seguimiento de las dislipidemias: tratamiento farmacológico con estatinas. Rev Colomb Cardiol. 2015;22:14-21.

9. [9] Ministerio de salud y Protección Social. Indicadores basicos 2013. Situación de salud en Colombia. 2014;1-104.

10. Allan GM, Nouri F, Korownyk C, Kolber MR, Vandermeer B, McCormack J. Agreement among cardiovascular disease risk calculators. Circulation. 2013;127:1948-56.

11. Fornasini M, Brotons C, Sellarès J, Martinez M, Galán ML, Sáenz I, et al. Consequences of using different methods to assess cardiovascular risk in primary care. Fam Pract. 2006;23:28-33.

12. González C, Rodilla E, Costa JA, Justicia J, Pascual JM. Comparación entre el algoritmo de Framingham y el de SCORE en el cálculo del riesgo cardiovascular en sujetos de 40-65 años. Med Clin (Barc). 2006;126:527-31.

13. Maiques Galán A, Antón García F, Franch Taix M, Albert Ros X, Aleixandre Martí E, Collado Gil A. Riesgo cardiovascular del SCORE comparado con el de Framingham. Consecuencias del cambio propuesto por las Sociedades Europeas. Med Clin (Barc). 2004; 123:681-5.

14. Barroso LC, Muro EC, Herrera ND, Ochoa GF, Hueros JIC, Buitrago F. Performance of the Framingham and SCORE cardiovascular risk prediction functions in a non-diabetic population of a
Spanish health care centre: a validation study. Scand J Prim Health Care. 2010;28(May 2009):242-8.

15. Marchant I, Boissel J-P, Kassaï B, Bejan T, Massol J, Vidal C, et al. SCORE should be preferred to Framingham to predict cardiovascular death in French population. Eur J Cardiovasc Prev Rehabil. 2009;16:609-15.

16. Eichler K, Puhan MA, Steurer J, Bachmann LM. Prediction of first coronary events with the Framingham score: A systematic review. Am Heart J. 2007;153, 722-31.e8.

17. Alcocer LA, Lozada O, Fanghänel G, Sánchez-reyes L, Camposfranco E. Estratificación del riesgo cardiovascular global. Comparación de los métodos Framingham y SCORE en población mexicana del estudio PRIT. Cir Cir. 2011;79:168-74.

18. Muntner P, Colantonio L, Cushman M, Goff D Jr, Howard V. Validation of the Atherosclerotic Cardiovascular Disease Pooled Cohort Risk Equations. JAMA. 2014;14:1406-15.

19. Ji K, Jang $Y$, Joo D, Oh B, Hoon S, Park S, et al. The ACC/AHA 2013 pooled cohort equations compared to a Korean Risk Prediction Model for atherosclerotic cardiovascular disease. Atherosclerosis. 2015;242:367-75.

20. Lee CH, Woo YC, Lam JKY, Fong CHY, Cheung BMY, Lam KSL, et al. Validation of the pooled cohort equations in a long-term cohort study of Hong Kong Chinese. J Clin Lipidol. 2015;9, 640-6.e2.

21. Bazo-alvarez JC, Quispe R, Peralta F, Poterico JA, Valle GA, Burroughs M, et al. Agreement between cardiovascular disease risk scores in resource-limited settings: evidence from 5 peruvian sites. Crit Pathways Cardiol. 2015;14:74-80.

22. Preiss D, Kristensen SL. The new pooled cohort equations risk calculator. Can J Cardiol. 2015;31:613-9. 